\title{
TITEL
}

Otto Lagodny

\section{Die Anrechnung ausländischer Haft nach $\ 51$ Abs. 4 Satz 2 StGB im Lichte der EMRK ${ }^{1}$}

Die jüngste Rechtsprechung des EGMR zum Verstoß der Haftverhältnisse in Russland gegen Art. 3 EMRK führt zu Anrechnungspflichten im Maßstab 1:3 bzw. 1:4 im Sinne von $\ 51$ Abs. 4 S. 2 StGB und zu weiteren Abstufungen. Dies ergibt eine Analyse der veröffentlichten deutschen Praxis.

Schlüsselwörter: Europäische Menschenrechtskonvention; Haftbedingungen; unmenschliche Behandlung; Anrechnung ausländischer Vorhaft

Title: Deduction of time spent in foreign prisons according to sec. 51 para. 4 sentence 2 of the German Code of Criminal Law

Abstract: Recent practice of the European Court of Human Rights concerning detention conditions in Russian prisons with regard to inhuman treatment (art. 3 ECHR) brings about a duty to deduct the time spent in foreign prisons under such conditions. The factor of deduction then is 1:3 or even 1:4. The factor has to be adjusted in a progressive way. Keywords: European Convention on Human Rights; prison conditions; inhuman treatment; deduction of time spent in a foreign prison.

Der EGMR hat jüngst grundlegende Aussagen zu den menschenrechtlichen Zuständen in russischen Haftanstalten sowie zur Berücksichtigung menschenwürdewidriger Haftumstände bei der Haftdauer gemacht (unten A). Das Urteil führt zu dogmatischen Erklärungen von $\ 51$ Abs. 4 S. 2 StGB. Diese sind deshalb Maßstab für die deutsche Praxis (unten B).

1 Dem Beitrag liegt ein Gutachten für die Kanzlei Dr. Schneider und Partner, Frankfurt am Main/ Köln, im Strafverfahren 5/28 KLs - 7470 Js 202783/12 (18/13) beim LG Frankfurt zugrunde. Das Landgericht hat eine siebeneinhalb Monate dauernde Auslieferungshaft in einem afrikanischen Staat mit dem Faktor 1:3 angerechnet. Dort ging es um einen Fall, in dem sich 50 Inhaftierte eine Zelle von $65 \mathrm{qm}$ teilen mussten (1,3 qm pro Person), nur eine Toilette und 4 Duschhähne hatten und die Zelle von Freitagabend bis Montagmorgen nicht verlassen konnten. Meinem Mitarbeiter, Herrn Mag. Patrick Bugelnig, danke ich sehr für tatkräftige Mithilfe bei der Erstellung der Druckfassung.

NK 26. Jg. 3/2014 


\section{A. Das Piloturteil zu menschenwürdewidrigen Haftumständen in Russland (Ananyev und andere)}

In einer Grundsatzentscheidung (Piloturteil) vom $10.01 .2012^{2}$ hat der EGMR insgesamt zur Frage Stellung genommen, unter welchen Voraussetzungen ein Verstoß der konkreten Haftbedingungen gegen Art. 3 EMRK in der Variante „unmenschliche und erniedrigende Behandlung" vorliegt. Anlass waren Individualbeschwerden zur Situation in russischen Haftanstalten.

Der Gerichtshof hat recht eindeutige Kriterien aufgestellt, wann ein Verstoß gegen Art. 3 EMRK und damit die Menschenwürde vorliegt. Er hat hervorgehoben, dass man aus den Berichten des Europäischen Antifolterkomitees eine Mindestgröße von 4 qm pro Person bzw. 3 qm entnehmen kann. Der EGMR betont, dass eine Größe von weniger als $3 \mathrm{qm}$ schon als solche und ohne weitere erschwerende Umstände zu einem Verstoß gegen Art. 3 EMRK führt. ${ }^{3}$ Die Tatsache, dass Insassen auf dem Boden schlafen müssen, wird dabei als erschwerender Umstand gewertet: ${ }^{4}$

Insgesamt hat der Gerichtshof folgende klare und eindeutige Regeln aufgestellt. ${ }^{5}$ Wenn nur eine dieser Voraussetzungen fehlt, liegt bereits ein Verstoß gegen das Verbot unmenschlicher und erniedrigender Behandlung nach Art. 3 EMRK vor:

- ein individueller Schlafplatz;

- 3 qm Grundfläche pro Insasse;

- Bewegungsfreiheit zwischen den Einrichtungsgegenständen.

Selbst wenn diese Regeln eingehalten sind, kann der Mangel an Frischluftzufuhr und natürlichem Licht in der Zelle zu einem Verstoß gegen Art. 3 EMRK führen. ${ }^{6}$ Darüber hinaus führen auch fehlende Möglichkeiten, täglich an der frischen Luft zu sein, zu einer Verletzung von Art. 3 EMRK. ${ }^{7}$ Im konkreten Fall hat der Gerichtshof einen Verstoß gegen Art. 3 EMRK eindeutig schon deshalb bejaht, weil die Zellen eine Grundfläche von $1,25 \mathrm{qm}$ pro Person ${ }^{8}$ bzw. weniger als $2 \mathrm{qm}$ pro Person ${ }^{9}$ aufwiesen.

Jüngst hat der Gerichtshof auch nochmals seine Praxis hervorgehoben, dass es für Art. 3 EMRK keiner Absicht der staatlichen Stellen bedarf, den Insassen erniedrigen zu wollen. ${ }^{10}$ Aus den vielen weiteren Entscheidungen zur Haftsituation im Lichte des

2 Ananyev und andere gegen Russland, Beschwerde Nrn. 42525/07 und 60800/08, rechtskräftiges Urteil der 1. Sektion. Siehe www.coe.int. In deutscher Übersetzung: NVwZ-RR 2013, 284, wobei die hier relevanten Passagen leider nicht enthalten sind.

3 No. 143-148 der Entscheidung.

4 No. 146 der Entscheidung.

5 No. 148 der Entscheidung.

6 Vgl. No. 149 und 153-155 der Entscheidung.

7 No. 150 der Entscheidung.

8 No. 131-133 der Entscheidung.

9 No. 138 der Entscheidung.

10 Torregiani u. a. gegen Italien, Urt. v. 08.01.2013, Kammer II, Beschwerde-Nr. 43.517/09; 46.822/09, 55.400/09, 57.875/09, 61.535/09, 35.315/10 und 37.818/10. Siehe auch die Nachweise bei Karpenstein/Mayer 2012 Art. 3 Rn. 8. 
Art. 3 EMRK sei auch noch auf den Fall Peers gegen Griechenland ${ }^{11}$ hingewiesen. Der EGMR hat einstimmig eine Verletzung von Art. 3 EMRK bejaht.

Auch das deutsche Bundesverfassungsgericht hatte sich mit einem Fall zu befassen, in dem die Frage eine Rolle spielte, ob bestimmte Haftbedingungen einen Verstoß gegen die Menschenwürde darstellen. Allerdings ging es im konkreten Verfahren um die (bejahte) Verletzung der Rechtsweggarantie nach Art. 19 Abs. 4 GG, so dass es keine Sachaussagen zu den Haftumständen gab. ${ }^{12}$ Hingewiesen sei auch auf eine ältere Entscheidung des BVerfG, ${ }^{13}$ in der ein Verstoß gegen die Menschenwürde bejaht wurde.

Im Rahmen eines Amtshaftungsverfahrens (hier: Prozesskostenhilfe) hat das BVerfG ${ }^{14}$ im Jahr 2011 hervorgehoben, dass als Faktoren für eine aus den räumlichen Haftbedingungen resultierende Verletzung der Menschenwürde in erster Linie folgende Faktoren maßgeblich sind:

„die Bodenfläche pro Gefangenem und die Situation der sanitären Anlagen, namentlich die Abtrennung und Belüftung der Toilette,[...], wobei als ein die Haftsituation abmildernder Faktor die Verkürzung der täglichen Einschlusszeiten berücksichtigt werden kann."15

\section{B. Dogmatische Konsequenzen des EGMR-Ansatzes für Deutschland}

Es stellt sich jetzt die Frage, welche Bedeutung die klaren Aussagen des EGMR für \51 Abs. 4 S. 2 StGB haben. Der EGMR hat zwei Möglichkeiten aufgezeigt (unten I). Für eine davon hat sich der deutsche Gesetzgeber entschieden. Sie soll dogmatisch analysiert werden. Dabei wird aufgezeigt, dass diese Anrechnungslösung einer menschenrechtlichen Pflicht entspricht (unten II). Vor diesem Hintergrund wird die deutsche Praxis insgesamt dargestellt (unten III).

I. Strafmilderung bzw. entsprechender Anrechnungsmaßstab als Kompensation im Sinne der EMRK

Im Piloturteil Ananyev and others hat der Gerichtshof hervorgehoben, dass jeder verurteilte Staat die Verletzung von Art. 3 EMRK anerkennen und dem Individuum eine unproblematisch verfügbare, effektive und vor allem unabhängig von einer Beschwerde zum EGMR verfügbare Möglichkeit der Kompensation eröffnen müsse. Wichtig ist in diesem Zusammenhang, dass es aus der Sicht des EGMR gleichgültig ist, ob die Kom-

11 Peers gegen Griechenland, Urt. v. 19.04.2001, Kammer II, Beschwerde-Nr. 28524/95.

12 BVerfG, Beschl. v. 13.03.2002 - 2 BvR 261/01 = NJW 2002, 2700 bzw. Beschl. v. 27.02.2002 2 BvR 553/01 = NJW 2002, 2699.

13 BVerfG, 2. Kammer des Zweiten Senats, Beschl. v. 16.03.1993 - 2 BvR 202/93 = NJW 1993, 3190.

14 BVerfG, 1. Kammer des Ersten Senats, Beschl. v. 22.02.2011 - 1 BvR 409/09 = NJW-RR 2011, 1043.

15 So die zusammenfassende Formulierung der NJW-RR-Redaktion.

NK 26. Jg. 3/2014 
pensation über eine Anrechnung wie in $\$ 51$ Abs. 4 StGB oder generell im Rahmen der Strafzumessung geschieht. ${ }^{16}$

Direkt hieran anschließend hat der Gerichtshof betont, dass eine Kompensation in Form einer Strafmilderung nur für einen Teil der russischen Gefangenen in Betracht komme, dann aber konsequent vorgenommen werden müsse. Vor allem müsse klar werden, welche Erwägungen des Gerichts dafür maßgeblich sind. Dieses „Messbarkeits“Erfordernis („measurability requirement“) setze die Individualisierbarkeit der Verletzung und ihrer Abhilfe voraus. Auch hierfür beruft sich der Gerichtshof auf eine ständige Praxis: ${ }^{17}$

Diesem Klarheitsgebot entspricht das deutsche Verfahren grundsätzlich, weil die Rechtsprechung deutlich macht, dass im ersten Schritt die deutsche Strafe als solche bestimmt werden muss und erst im zweiten Schritt die ausländische in ihrer geschätzten Belastung abgezogen werden muss. ${ }^{18}$

\section{Konsequenzen dieses Ansatzes für $\ 51 \mathrm{Abs} .4 \mathrm{StGB}$}

Vor dem Hintergrund dieser Erklärung geht es bei dem Anrechnungsfaktor nach $\$ 51$ Abs. 4 S. 2 StGB um eine menschenrechtlich zwingende Konsequenz. Denn die deutschen Organe der Strafrechtspflege sind objektiv-rechtlich mitverantwortlich für die Verletzung der Menschenwürde. Allerdings hat das Bundesverfassungsgericht in einer Entscheidung aus dem Jahr $1981^{19}$ entschieden, dass ein deutsches Auslieferungsersuchen als solches den Beschwerdeführer nicht unmittelbar in seinem Grundrecht aus Art. 2 Abs. 2 Satz 2, 104 Abs. 1 Satz 1 GG verletzt. Aber aus dieser Entscheidung kann man schon deshalb keine Konsequenzen für den Umrechnungsfaktor herleiten, weil es im Jahr 1981 um eine völlig andere grundrechtliche Frage ging, nämlich darum, ob bereits ein Auslieferungsersuchen als solches einen Grundrechtseingriff bewirkt, weil das Ersuchen auch zur Folge haben kann, dass der Verfolgte im ersuchenden Staat in Auslieferungshaft genommen wird. Im Anwendungsbereich des $\$ 51$ Abs. 4 S. 2 StGB geht es jedoch um etwas gänzlich anderes, nämlich darum, wie es rechtlich zu berücksichtigen ist, dass sich der Verfolgte im ersuchten ausländischen Staat tatsächlich in Auslieferungshaft befand und unter Umständen vollzogen worden ist, die eine eklatanten Verstoß gegen Art. 3 EMRK und damit den europäischen Mindeststandard darstellen bzw. darstellen würden, wenn der ersuchte Staat nicht die EMRK ratifiziert hat.

Es kommt keinesfalls darauf an, ob die deutschen Behörden mit der Absicht gehandelt hätten, den Verfolgten zu erniedrigen. Das betont der EGMR aus- und nachdrücklich. ${ }^{20}$ Das Bundesverfassungsgericht und generell die deutsche Rechtsprechung folgt einer nationalen und vor allem auch internationalen Entwicklung, die im Jahr 1989 mit

16 No. 221-231 der Entscheidung.

17 No. 225 der Entscheidung.

18 Siehe oben A II.

19 BVerfG, Beschl. v. 25.03.1981 - 2 BvR 1258/79 = BVerfGE 57, 9.

20 No. 117 der Entscheidung. 
der Soering-Entscheidung des EGMR eingesetzt hat und bis heute anhält. ${ }^{21}$ Als generelle Aussage dieser Rechtsprechung kann man formulieren: Die grundrechtliche Verantwortlichkeit des einen Staates endet nicht deshalb, weil die Folgen des Handelns dieses Staates im Ausland eintreten. ${ }^{22}$ Daran ändert sich auch für die konkrete Frage der Anrechnung von Auslieferungshaft dadurch nichts, dass sich den deutschen Strafverfolgungsbehörden vielfach keine Alternative bietet, als einen international flüchtigen mutmaßlichen Straftäter zur Fahndung auszuschreiben mit der Folge, dass er auch in einem Staat festgenommen wird, dessen Haftanstalten - wie hier - keinen europäischen Maßstäben standhalten. Wie jedoch bereits die deutsche Rechtsprechung betont, geht es nicht an, den Verfolgten auch noch dafür verantwortlich zu machen, weil er sich freiwillig gerade in einen solchen Staat begeben habe. ${ }^{23}$

Es sei insbesondere hingewiesen auf die Argumentation des BVerfG in der Entscheidung vom 03.09.2009 zur dogmatischen Figur der „Substitution“ einer inländischen Prozesshandlung durch eine ausländische Prozesshandlung. Sie wird für die Frage, ob griechische Prozesshandlungen als verjährungsunterbrechend im Sinne des deutschen Strafrechts $(\$ 78 \mathrm{c}$ StGB) anerkannt werden können, vorgenommen mit der Folge, dass die Tat im Hinblick auf das deutsche Auslieferungsrecht ( $\$ 9$ Nr. 1 IRG) noch als nicht verjährt angesehen werden kann. Aus dieser und aus weiteren Konstruktionen, die hinter den Entscheidungen liegen, lässt sich der Grundsatz ableiten: „Die Erfüllung der in beiden Staaten nach jeweils nationalem Recht bestehenden Normvoraussetzung X (z. B.

21 Vgl. Schomburg/Lagodny/Gleß/Hackner 2012 (nachfolgend: IRhSt-Bearbeiter), Einleitung Rn. 11 (Leitentscheidungen zur Dreidimensionalität); Schomburg/Lagodny NJW 2012, 348: 26.01.1982 - BVerfGE 59, 280 = NJW 1982, 1214 (Abwesenheitsurteil); 13.01.1987 - BGHSt 34, 256 = NJW 1988, 655 (Zusicherung der Nichtverhängung der Todesstrafe ist vom OLG nicht nur von der Bewilligungsbehörde - zu prüfen); 07.07.1989 - EGMR im Fall Soering, z. B. NJW 1990, 2183 (Verantwortlichkeit eines EMRK-Staates für Konsequenzen seines Handelns außerhalb des Territoriums von EMRK-Staaten) und in der Folge die weitere Praxis des EGMR zu Auslieferungs- und Abschiebehindernissen aus Art.3 EMRK; 18.06.1997 BVerfGE 96, 100 = NJW 1997, 3013 (grundrechtliche Absicherung der Ermessenkontrolle für Frage, ob ein Ersuchen auf Urteilsvollstreckung an ausländischen Staat gestellt wird); 26.06.2001 - IGH im Fall La Grand (Germany v. United States of America), IGH v. 27.06.2001 = EuGRZ 2001, 287, mit nationaler Folgerechtsprechung zur Benachrichtigungspflicht nach Art. 36 WÜK bei Festnahme eines ausländischen Staatsangehörigen; 18.07.2005-BVerfG NJW 2005, 2289 zum ersten Gesetz zum Europäischen Haftbefehl (Berücksichtigung von Grundrechtsbelangen durch den Gesetzgeber; kein Ausschluss der Anfechtbarkeit von Bewilligungsentscheidungen); 03.09.2009 - BVerfG (2 BvR 1826/09) StraFO 2009, 455 = NJOZ 2010, 1436) und 09.10.2009 (2 BvR 2115/09 = StraFO 2009, 458) jeweils BVerfG zum Rechtsschutz bei der Auslieferung eines deutschen Staatsangehörigen; 16.09.2010 - BVerfG, Beschl. v. 16.09.2010 2 BvR 1608/07 (zur Garantie des Art. 104 GG im Auslieferungshaftverfahren) = StraFo 10, 495 $=$ NJOZ 2010, 1431 .

22 So bereits BVerfGE 6, 294, 295.

23 „Es ist ermessensfehlerhaft bei der Bestimmung des Maßstabes der Anrechnung im Ausland verbüßter Haft nach $\$ 51$ IV StGB darauf abzustellen, daß ein Verurteilter sich strengere Haftbedingungen im Ausland wegen seiner Flucht selbst zuzuschreiben hat." (OLG Celle, Beschl. v. 02.07.1997 - 3 Ws 131/97 (II) = NStZ 1998, 137).

NK 26. Jg. 3/2014 
„Strafverfahren“) im Staat 1 wird sinngemäß so behandelt, als sei sie auch die Erfüllung der Normvoraussetzung im Staat 2 “. ${ }^{24}$

Das bedeutet konkret: Eine Rechtsordnung, die sich für eine Anrechnungslösung und in der Konsequenz: für eine Anrechnung mit höherem Maßstab bei problematischen bis menschenrechtswidrigen Haftverhältnissen im ausländischen Staat - entschieden hat, muss aus Gründen menschenrechtlicher Verantwortlichkeit eine Kompensation eines Verstoßes gegen Art. 3 EMRK vornehmen. Dieser Verstoß kommt auch und gerade dann in Betracht, wenn er für die Behörden des ersuchenden Staates unvermeidbar zur Realisierung seiner Strafverfolgungsverpflichtung war und auch völlig unbeabsichtigt. Der Verstoß muss aber hinreichend kompensiert werden. Wenn aber die Fälle des Art. 3 EMRK kompensiert werden müssen, dann leuchtete es nicht ein, wenn für alle anderen Fälle keinerlei Kompensation erfolgte. Eine solche „Alles-oder-Nichts"-Lösung wäre im Hinblick auf das Gleichheitsgrundrecht (Art. 3 Abs. 1 GG) wenig überzeugend. Vor diesem Hintergrund ist die Abstufungslösung der deutschen Praxis rückblickend zu erklären.

\section{Deutsche Praxis zu $\ 51$ StGB}

\section{Ausgangspunkt}

In einer Entscheidung aus dem Jahre 1981 hat der BGH zu $\ 51$ Abs. 4 Satz 2 StGB grundsätzlich ausgeführt, ${ }^{25}$ was auch bis heute der Praxis zugrunde liegt: ${ }^{26}$

„Die Vorschrift des $\int 51$ Abs. 4 Satz 2 St GB, wonach der Richter, wenn er ausländische Strafe auf deutsche Strafe anrechnet, den Maßstab nach seinem Ermessen bestimmt, berubt auf der Rechtsmeinung, die sich zu $\int 7$ StGB in der bis 31. August 1969 geltenden Fassung gebildet hatte (RGSt 35, 41; Niethammer bei Olshausen, StGB, 12. Aufl., $\int 7$ Anm. 4). Ausgeübt wird das Ermessen durch, Schätzung des im Ausland erlittenen Strafübels und Umsetzung desselben in ein dem inländischen Strafsystem zu entnebmendes Äquivalent' (RG a.a.O. S. 43). Der Richter hat zu erwägen, wie schwer das Übel wiegt, das dem Verurteilten durch den ausländischen Strafvollzug widerfabren ist, und wieviel dieses Übel von demjenigen schon vorweggenommen hat, mit dem das inländische Urteil den Verurteilten belasten will; dabei ist der Maßstab zu berücksichtigen, der sich aus dem Vergleich der ausländischen mit der inländischen Strafenordnung ergibt' (Niethammer a.a.O.)."

Entscheidend ist danach ein Vorgehen in zwei aufeinander folgenden Schritten: Zunächst wird die deutsche Strafe bestimmt. Dann erst wird die ausländische geschätzt und abgewogen. Entscheidend ist bei diesem zweiten Schritt die Schätzung und Erwägung der

24 RhSt - Schomburg et al. 2012, Einl. Rn. 129.

25 BGH, Beschl. v. 08.12.1981 - 1 StR 648/81 = BGHSt 30, 282, Rn. 3 (in der von Jurion.de veröffentlichten Fassung).

26 BGH, Beschl. v. 28.01.1986 - 1 StR 652/85 = NStZ 1986, 312. 
durch den ausländischen Vollzug vorweggenommenen Übelszufügung, die eigentlich erst durch das deutsche Urteil erfolgen soll.

Der BGH rekurriert damit auf eine über hundert Jahre alte Entscheidung des Reichsgerichts aus dem Jahre $1901^{27}$ und einen Kommentar aus dem Jahre 1942. Wichtig ist in diesem Zusammenhang, dass die Entscheidung des Reichsgerichts ebenso wie die Literatur dieser Zeit zwar eine „Ermessenentscheidung“ des deutschen Strafgerichts forderte, dabei aber überhaupt nicht ausländische Haftbedingungen im Auge hatte, die unter Umständen gravierender sind. Vielmehr ging es nur um die abstrakt-generelle Verschiedenheit von Strafarten in den jeweiligen Staaten: „Selbstverständlich setzt die Anrechnung [...] Kommensurabilität ${ }^{28}$ der Strafen voraus. Da inländische und ausländische Strafen selten völlig gleichwertig sein werden, so bleibt dem richterlichen Ermessen ein weiter Spielraum.“"29 Wenn kein „über die Verschiedenheit der Bezeichnung hinausgehender Unterschied vorhanden ist, so ist damit die Anrechnung vorgezeichnet. Fehlt es hieran, so hat der inländische Richter die Art der Anrechnung nach seinem Ermessen [...] zu bestimmen." ${ }^{30}$

Vor diesem Hintergrund wurde zugleich schlüssig erklärt, warum es seinerzeit keine rechtliche Bindung gegeben haben kann: Es ist nämlich für den (nationalen) Gesetzgeber „unmöglich [...], sämtliche aus- und inländischen Strafarten zueinander ins Verhältnis zu setzen.“31

Der BGH hat damit die Vorschrift des $₫ 51$ Abs. 4 Satz 2 StGB auf der Grundlage der Rechtsprechung und Lehre zu $\$ 7$ StGB a. F. weiterentwickelt. Im Jahre 1981 hat er allgemein den Weg eröffnet, das neue Problem unterschiedlicher Haftbedingungen über einen erhöhten Anrechnungsmaßstab zunächst mit dem Gleichheitsgrundrecht in Einklang zu bringen: Durch Anrechnung der ausländischen Haft mit einem gegebenenfalls günstigeren abstrakt-generellen Maßstab (ein Tag im Staat X wird gewertet wie 2, 3 oder mehr Tage in Deutschland). Eine „Strafzumessungslösung“ gibt es als anderen Weg z. B. in Österreich. Dort wird der im Einzelfall härtere und belastendere Vollzug von Verfahrenshaft ausschließlich bereits bei der Strafzumessung strafmildernd berücksichtigt. ${ }^{32}$ Dort gibt es die zwei Schritte nicht.

\section{Charakterisierung der nachfolgenden Praxis}

Der Ansatz des BGH wurde in der Praxis zunächst eher zurückhaltend umgesetzt. So hatte etwa das LG Landau ${ }^{33}$ schon im Jahr 1980 einen Maßstab von 1:1,5 angenommen für sehr harte Bedingungen im Libanon (zahlreiche Mitgefangene in der Zelle, schlechte

27 Urt. v. 17.12.1901 g. St. Rep. 4697/01 = RGSt 35, 41.

28 Hervorhebung im Original.

29 Frank 1931. Ebenso: von Olshausen 1914, $\$ 7$ Anm. 4.

30 Schwartz 1914, Anm. 7.

31 So schon von Olshausen 1892, Anm. 5.

32 Höpfel/Kathrein \62-67 StGB, in: Höpfel/Ratz 2011, \66 Rn. 2 unter Hinweis auf die einschlägige Entscheidung des OGH in der Rechtssache 11 Os 85/95.

33 LG Landau, Urt. v. 28.08.1980 - 21 Js 719/79 KLs = NStZ 1981, 64.

NK 26. Jg. $3 / 2014$ 
Behandlung durch Aufsichtspersonal, schlechte Verpflegung und ärztliche Betreuung). Diese Bedingungen werden zudem nicht sehr genau spezifiziert.

Das OLG Frankfurt/Main hat im Jahr 1987 die Belegung einer Einmann-Zelle in Italien mit sechs Personen bei großer Hitze, Ungeziefer und sonstigen sehr unhygienischen Verhältnissen mit dem Maßstab 1:2 berücksichtigt. ${ }^{34}$

Auf der Grundlage dieses Schätzungs- und Erwägungsansatzes hat sich in den über dreißig Jahren seit dieser Grundsatzentscheidung des BGH eine doch bedeutsame Praxis der deutschen Strafgerichte entwickelt. Der Maßstab 1:1 wird seit einer Grundsatzentscheidung des BGH aus dem Jahre 2011 grundsätzlich für EU-Staaten herangezogen. ${ }^{35}$ Der Maßstab von 1:2 wurde - mit gelegentlichen Modifikationen von 1:1,5 bis 1:2,5 relativ häufig angenommen. In nicht wenigen Entscheidungen wird jedoch auch ein Umrechnungsmaßstab von 1:3 zugrunde gelegt.

Insgesamt war eine Vielzahl von Kriterien maßgeblich, die sich wie folgt strukturieren lassen: ${ }^{36}$

- Haftraum (Größe etc.) und Hygiene (v. a. Toilette)

- Misshandlungen

- Mitgefangene (z. B. aggressiv; ansteckend erkrankt; etc.)

- Vollzugspersonal

- Ärztliche und medizinische Betreuung

- Essen und Trinken

- Beleuchtung

- Hofgang

- Kontakt nach außen

- Möglichkeit zur Arbeit

- Information/Post

Zusammenfassend kann man insoweit allerdings nur sagen: „Je mehr Mängel vorliegen, umso eher wird das Gericht zu einem vorteilhafteren Anrechnungsmaßstab gelan-

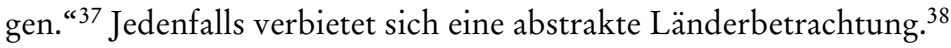

34 OLG Frankfurt/Main, Beschl. v. 02.02.1987 - 3 Ws 559/86 = StV 1988, 20.

35 BGH, Urt. v. 27.01.2011 - 4 StR 502/10 = NStZ 2011, 699 = StV 2011, 412.

$36 \mathrm{Vgl}$. Heintschel-Heinegg 2012, $551 \mathrm{Rn} .17 \mathrm{a}$.

37 So ausdrücklich Heintschel-Heinnegg 2012, $\$ 51$ Rn. 17a. Er entwickelt hieraus einen für die Strafverteidigung sehr nützlichen Katalog von Fragen an den Mandanten. $\mathrm{Zu}$ beachten ist nämlich: Die Darstellung des Verfolgten muss „im Zweifel“ zugrunde gelegt werden: BGH, Beschl. v. 12.09.2000 - 4 StR 299/00; vgl. auch BGH, Beschl. v. 08.07.2004 - 5 StR 151/04; BGH, Beschl. v. 13.08.2009 - 3 StR 255/09 = NStZ-RR 2009, 370: 2:1 Australien. Bei allgemeinen Ausführungen des Auswärtigen Amtes, wonach bei einer Untersuchung der konkreten ausländischen Justizvollzugsanstalt keine gravierenden Mängel festgestellt werden konnten, reicht sogar die konkrete Auflistung und Beschreibung der Haftumstände nicht aus, um die [konkrete] Schilderung des Angeklagten von den tatsächlichen Bedingungen zu widerlegen. Dies gilt vor allem für Punkte, welche zwar der Verurteilte, nicht aber das Auswärtige Amt erwähnt, wie hier konkret z. B. die hygienischen Bedingungen der JVA oder die tatsächliche Erreichbarkeit von medizinischem Personal (LG Verden an der Aller, Urt. v. 06.07.2006 - 2-1/06 = StV 2007, 362: 1:3 Mazedonien).

38 Kett-Straub in: Kindhäuser/Neumann/Paeffgen 2013, \51 Rn. 40. 
Allerdings lassen sich der veröffentlichten Rechtsprechung folgende Tendenzen entnehmen, wenn man abstrakt-generell nach den Staaten und deren geo-politischer Lage gliedert:

Der Maßstab 1:2 wurde vornehmlich auf andere europäische Staaten angewendet sowie auf Staaten mit westlichem Wirtschaftsstandard außerhalb von Europa. Dabei ist anzumerken, dass einige der dort aufgeführten Fälle aus den 80er und 90er Jahren des 20. Jahrhunderts in Frankreich, Italien und Spanien stammen. Wie noch aufzuzeigen sein wird, kommt die Schilderung der Haftumstände in diesen Fällen denjenigen nahe, für die heute ohne weiteres der Maßstab 1:3 zugrunde gelegt wird.

Der Maßstab 1:2 wurde zwar auch auf außereuropäische und nicht-westliche Staaten angewendet. Oft finden sich aus prozessualen Gründen in den Entscheidungen aber keine näheren Sachverhaltsangaben zu den Haftumständen. ${ }^{39}$ Deshalb ist ein Vergleich etwa mit heutigen Standards nicht möglich.

Der Maßstab von 1:3 kam in der Regel bei außereuropäischen nicht-westlichen Staaten zur Anwendung. Unter den anderen Staaten (unten: „sonstige“) ging es in 3 der nachgewiesenen 5 Entscheidungen um Fälle aus Spanien in den 90er-Jahren des 20. Jahrhunderts.

3. Tabellarische Übersicht zur Praxis im Einzelnen

\section{Maßstab 1:2 (jeweils zeitlich geordnet)}

\section{Europa}

1:2 Spanien (BGH, Urteil vom 11.07.1985 - 4 StR 293/85 = NStZ 1985, 497)

1:2 Italien (OLG Frankfurt/Main, Beschluss vom 02.02.1987 - 3 Ws 559/86 = StV 1988, 20)

- Einmann-Zelle in Italien mit sechs Personen bei großer Hitze

1:2 Spanien (LG Zweibrücken, Beschl. v. 30.10.1987 - 2 StVK 606/87 = NStZ 1988, $71=$ NStE $\int 51$ StGB Nr. 3)

- für 1 Monat mit 60 Häftlingen in einem 20 m langen und 6-8 Meter breiten Raum, 1:2 als „EU-Bonus“ 2 Jahre nach dem EU-Beitritt?

1:1,5 Frankreich (LG Essen, Beschl. v. 15.10.1990 - 32 StVK 1075/90 = StV 1991, 170)

- Gefängnis von Montpellier

1:2 Spanien (OLG München, Beschl. v. 03.09.1993 - 2 Ws 1048/93 = NStE \51 StGB Nr. 22)

1:2 Spanien (LG Kleve, Urt. v. 21.09.1994 - 1 KLs 41/94 = NStZ 1995, 192)

1:2 Portugal (BGH, Beschl. v. 18.10.1994 - 1 StR 564/94 = NStZ-RR 1994, 30) ${ }^{40}$

1:1,5 Frankreich (LG Hamburg, Beschl. v. 18.06.1996-609 StVK 1070/89 = StV 1997, 87)

- St. Joseph Gefängnis in Lyon

39 Vgl. z. B. BGH, Beschl. v. 27.11.2003 - 3 StR 221/03, wo es nur darum ging, dass die nicht mitgeteilten Erwägungen des Instanzgerichts auch im Tenor zum Ausdruck kommen müssen.

40 Zitiert bei Satzer/Schmitt/Widmaier 2009 (nachfolgend: SSW-Autor), $\$ 51$ Rn. 32.

NK 26. Jg. $3 / 2014$ 
1:2 Türkei (LG Weiden, Urt. v. 01.10.1997 - KLs Js 9227/94 = NStZ-RR 1999, 30)

1:2 Schottland (BGH, Beschl. v. 21.09.1999 - 5 StR 416/99 = wistra 1999, 463)

- wegen Krankheit des in Schottland Verurteilten höher als 1:1

1:2 Portugal (BGH, Beschl. v. 20.05.2003 - 15 StR 170/03) ${ }^{41}$

1:2 Estland (BGH, Beschl. v. 27.11.2003 - 3 StR 221/03)

- nur „Urteilstenor“ anzupassen; keine Sachaussagen Außer-Europa und westlich

1:2 Australien (LG Heilbronn, Urt. v. 21.10.1991 - I StVK 363/90 = StV 1992, 429)

- australische „in etwa“ mit den spanischen Haftbedingungen zu vergleichen

1:2 Slovakei (LG Kleve, Beschl. v. 26.11.2002 - 2 StVK 393/02 = StV 2003, $453=$ StraFO 2003, 281)

1:2 Australien (BGH, Beschl. v. 13.08.2009 - 3 StR 255/09 = NStZ-RR 2009, 370)

Außer Europa und nicht westlich

1:1,5 Libanon (LG Landau, Urt. v. 28.08.1980 - 21 Js 719/79 KLs = NStZ 1981, 64). 1:2 Marokko (OLG Zweibrücken, Beschl. v. 27.05.1992 - 1 Ws 241/92-242/92 = BeckRS 1992, $09618=$ GA 1993, $126=$ NStE $\ 51$ StGB Nr. 21) - dazu näher unten D II 2.

1:2 Paraguay (LG Zweibrücken, Urt. v. 17.08.1994 - 424 Js 3601/92 1 KLsMDR 1995, $84=$ NStE $\int 51$ StGB Nr. 25)

- Zellen mit 3-4 Insassen ohne Trennung zwischen Untersuchungs- und Strafhaft; belastende klimatische Verhältnisse, ungenügend sanitäre Einrichtungen, mangelhafte Versorgungslage. Angeklagter konnte sich zusätzliches Essen und Bewegungsfreiheit erkaufen.

1:2 Brasilien (LG Oldenburg, Urt. v. 30.06.1999 - $2 \mathrm{KLs} 3 / 99=\mathrm{StV} 2000$, 86)

1:2 Thailand (AG Schwäbisch Hall, Urt. v. 04.10.2000 - 1 Ls 43 Js 44778/97 = StrFO 2001, 246)

- Die Festsetzung des Maßstabs von 1:2 bei durchaus sehr schweren Haftbedingungen erfolgte auch deshalb, weil das Gericht bedacht habe, dass sich der Verurteilte in Kenntnis der Strafverfolgung nach Thailand „abgesetzt“ habe. Diese unzulässige Erwägung ${ }^{42}$ macht diese Entscheidung nicht verwertbar.

1:2 Mexiko (BGH, Beschl. v. 14.05.2002 - 5 StR 157/02)

- kein Ermessenfehler für die Quote von 1:2 für Mexiko, aber ohne Angabe zu den maßgeblichen Umständen; wobei aber die Umstände der Freiheitsentziehung auch strafmildernd berücksichtigt worden sind.

1:1,5 Serbien (LG München I, Urt. v. 22.10.2009 - 21 Ns 367 Js 40382/06 = StV 2010, 309)

1:2 Weißrussland (LG Ulm, Urt. v. 12.05.2010 - 1 KLs 14 Js 24481/07 = StV 2010, 527)

41 Zitiert bei SSW-Eschelbach, $₫ 51$ Rn. 32.

42 Siehe OLG Celle, Beschl. v. 02.07.1997 - 3 Ws 131/97 (II) = NStZ 1998, 137. 
1:2 Malaysia (BGH, Beschl. v. 13.07.2011 - 2 StR 179/11)

- Mangels konkreten Anhaltspunkten für eine „größere Belastung“

1:1,5 Russland (BGH, Beschl. v. 07.01.2009 - 5 StR 490/08)

- nur Faktor 1,5, weil Auslieferungshaft in einem „seiner Heimat zumindest ähnlichen Kulturkreis vollzogen" wurde.

Maßstab 1:3 (jeweils zeitlich geordnet)

Außereuropäisch und nicht westlich

1:3 Kamerun (LG Köln, Urt. v. 17.12.1991 - 112 - 37/91 = NStE $\ 51$ StGB Nr. 20)

1:3 Marokko (AG Bremen, Beschl. v. 27.03.1992 - 74 Gs 199/92 = StV, 1992, 428 mit redaktionellem Leitsatz mit der Angabe des Maßstabs von 1:3)

1:3 Kenia (OLG Zweibrücken, Urt. v. 10.09.1996-412 Js 95 18/93-1 Kls jug = NStZRR 1997, 206 = MDR 1997, 279)

1:2,5 Brasilien (LG München II, Urt. v. 27.01.2000 - 1 KLs 48 Js 39789/95 = StV 2001, 19)

- 9 qm Zellenfläche mit 14-17 Mitinsassen belegt; Loch im Boden als Toilette; fensterlose Zelle ohne Frischluft; Temperatur zeitweise bei 40 Grad Celsius.

1:2,5 Pakistan (LG Nürnberg-Fürth, Urt. v. 09.01.2002 - 1 KLs 359 Js 21203/99 = StV 2002, 606)

1:3 Dominikanische Republik (LG Chemnitz, Urt. v. 21.12.2005 - 1 Ks 200 Js $14299 / 03$ = StV 2007, 138)

- Einzelumstände werden nicht genannt.

1:3/1:2 Ecuador (BGH, Beschl. v. 08.07.2004 - 5 StR 151/04 = StraFO 2004, 391)

1:3 Thailand (OLG Karlsruhe, Beschl. v. 13.01.1998 - 1 Ws 314/97, Die Justiz 1998, 570-571)

- Einzelumstände werden nicht genannt.

\section{Sonstige}

1:3 Spanien (LG Bremen, Urt. v. 13.12.1991 - 14 KLs 501 Js 6582/89= StV 1992, 326)

- Misshandlungen von Mitgefangenen und von Wachpersonal, Sammelzelle für ca. 100 Personen ohne Tisch und ohne Stuhl; Holzverschläge ohne Matratzen als Betten. Eine einzige „Toilette“ in Form eines in den Boden eingelassenen Lochs; Ungeziefer (Kakerlaken, Wanzen).

1:3 Spanien (OLG Düsseldorf, Beschl. v. 30.05.1995 - 4 Ws 94/95 = StV 1995, 426)

- „katastrophale Zustände“

1:3 Bulgarien (LG Berlin, Urt. v. 06.05.1997 - [530] 69 Js 207/94 KLs [87/96] = StV 1998,347

1:3 Spanien (BGH, Beschl. v. 12.09.2000 - 4 StR 299/00)

- 1:3 zum Ausschluss jeder Benachteiligung ${ }^{43}$

1:3 Mazedonien (LG Verden an der Aller, Urt. v. 06.07.2006-2-1/06 = StV 2007, 362)

- 3 qm Raum für 2-3 Häftlinge, kein Zugang für Frischluft

43 Vgl. auch BGH, Beschl. v. 07.01.1998 - 2 StR 652/97.

NK 26. Jg. 3/2014 
1:3 Kamerun (LG Köln, Urt. v. 17.12.1991 - 112 - 37/91 = NStE $\int 51$ StGB Nr. 20)

Diese Entscheidung deutet an, dass auch ein höherer Maßstab als 1:3 in Betracht gekommen wäre:

„Mit körperlichen Mißhandlungen verbundene Vernebmungsmethoden der örtlichen Polizei; Unterbringung , in einer kargen, fensterlosen Betonzelle mit Ungeziefer, obne ordentliche sanitäre Einrichtungen, obne Schlafstellen, obne Verpflegung, obne die grundsätzliche Möglichkeit eines Freigangs sowie schließlich der Zusammenlegung von bis zu mebr als 100 Häftlingen. Auf die Person des Angeklagten bezogen war jedoch andererseits zu berücksichtigen, daß dieser in seiner besonderen Situation als Europäer unter ansonsten afrikanischen Mithäftlingen in der Lage gewesen ist, den schweren Haftbedingungen besser zu begegnen als seine afrikanischen Mithäftlinge. Aufgrund seiner französischen Sprachkenntnisse, seinen von der Haft gewonnenen Beziehungen zu einem nach seiner Schilderung nicht unbedeutenden einheimischen Personenkreis, u. a. zu dem Generalstaatsanwalt sowie aufgrund seiner Betreunng durch die deutsche Botschaft hat der Angekl. die äußerst widrigen Haftumstände für sich etwas abmildern können, was auch in der von ibm geschilderten Rolle eines sog. Zellenchefs zum Tragen gekommen ist. In diesem Zusammenhang kann letztlich auch nicht überseben werden, daß der Angekl. ungeachtet des sicher sebr gravierenden Hafterlebnisses für die Zukunft eine Rückkebr nach Kamerun nicht ausschließen will. Aufgrund der insgesamt der Kammer zur Verfügung gestandenen Erkenntnisse erscheint zum Ausgleich ungleich härterer Haftbedingungen eine Anrechnung der ausländischen Haft in dem angeordneten Maßstab geboten und angemessen."

1:3 Kenia (OLG Zweibrücken, Urt. v. 10.09.1996 - 412 Js 95 18/93 - 1 Kls jug = NStZRR 1997, 206)

In der Entscheidung werden folgende Zustände geschildert, um bereits im Jahr 1996 zum Maßstab 1:3 zu gelangen:

„... Wäbrend seiner Auslieferungshaft in Kenia vom 09.08.1995 bis zum 08.12.1995 befand sich der Angekl. mit Ausnabme der beiden letzten Wochen im Zentralgefängnis von Mombasa. Dort war er in einem 50 qm großen Raum als einer von 80 Gefangenen untergebracht. Die Gefangenen mußten auf dem Boden schlafen. Eine Körperhygiene fand nicht statt. Als Toilette stand ein Loch im Boden in der Mitte des Raumes zur Verfügung. [...] Der Angekl. erkrankte wäbrend der Haftzeit an Malaria, Rubr und Hautkrankheiten. In den beiden letzten Wochen befand er sich im Polizeigefängnis. Dort waren 8 Männer, einige Frauen und auch Kinder in einer Zelle untergebracht. Es bestand eine Duschmöglichkeit im Hof. Auch in dieser Zeit hatte der Angekl. keine Möglichkeit, die Kleidung zu wechseln. [...]“ 
1:2,5 Pakistan (LG Nürnberg-Fürth, Urt. v. 09.01.2002 - 1 KLs 359 Js 21203/99 = StV 2002, 606)

„Die Angekl. [...] lebten [...] mit weiteren 4 bis 6, nur ausländischen-darunter einer italienischen - Gefangenen in einer gesonderten Zelle, welche sich in einer Baracke befand. Die Baracke selbst war mit 70 bis 80 Franen und Kindern vorwiegend pakistanischer Staatsangehörigkeit belegt.

Die Zelle hatte keine Betten. Die Angekl. mußten auf dem Steinboden schlafen. Die Zelle hatte ein kleines Fenster. Als Toilette diente ein Loch im Boden. Die Angekl. konnten sich die meiste Zeit außerhalb ibrer Zelle frei in der Baracke bewegen. Zudem hatten sie die Möglichkeit, den Gefängnishof zu betreten. Die Zellentür wurde nur selten geschlossen. Die Angekl. mußten sich zunächst selbst Decken kaufen. Auch Wasser und Verpflegung mußten sie von der Kantine der Haftanstalt erwerben. Sie konnten dort europäisches Essen kaufen. Das Geld dazu bekamen sie über die Deutsche Botschaft. Von dort bekamen sie auch Bücher.

Die Angekl. wurden monatlich von einem Angehörigen der Deutschen Botschaft besucht, der ibnen jew. Geld übergab, das von den Eltern der Angekl. kam. Die Angekl. konnten brieflich nach außen kommunizieren. Aufgrund Bestechung des Gefängnispersonals gelang es ibnen, Briefe aus dem Gefängnis hinauszuschmuggeln. Die bygienischen Verbältnisse in der Baracke waren schlecht. Die pakistanischen Strafhäftlinge warfen Müll und Essensreste in der Baracke einfach in die Ecken. Auch im Hof lagen Essensreste herum. In ibrer Zelle hatte die Angekl. Ph. dafür gesorgt, daß Essensreste und sonstiger Abfall dort nicht verblieb, sondern nach außen geschafft wurde. Wegen der Essensabfälle gab es im Gefängnis u.a. Ratten, Kakerlaken und anderes Getier. Die Angekl. wurden vom Gefängnispersonal weder geschlagen noch wurde ibnen sonst Gewalt angetan. Sie bekamen aber mit, daß die pakistanischen Gefangenen oftmals geschlagen wurden. Zudem blieb ibnen - akustisch durch das Herunterklappen der Falltür - nicht verborgen, daß wäbrend ibres Gefängnisaufenthalts mebrfach Hinrichtungen vollzogen wurden.

Eine medizinische Versorgung fand offensichtlich nur bei ernsten Krankbeiten und lautstarker Intervention der Betroffenen statt. [...]

1:3 Bulgarien (LG Berlin, Urt. v. 06.05.1997 - (530) 69 Js 207/94 KLs (87/96) = StV 1998, 347)

Das LG Berlin ging von folgendem Sachverhalt aus, um im Jahr $1997 \mathrm{zu}$ einem Anrechnungsmaßstab von 1:3 für die Anrechnung auf Jugendstrafe zu gelangen:

„Die Haftbedingungen waren im Vergleich zu den Verbältnissen in deutschen Strafanstalten schwer. Im Untersuchungsgefängnis mußte sich der Angekl. eine sechs Quadratmeter große fensterlose Zelle mit zwei anderen erwachsenen Gefangenen teilen. In der Regel konnten sie diese Zelle für eine Stunde am Tag verlassen; sie wurden dann in einen größeren Raum gefübrt, in dem eine unbrauchbare Tischtennisplatte stand. 
Als Abort diente ein in der Zelle aufgestellter Eimer. Ein 5 l-Kanister mit Wasser mußte den Tag über zum Trinken und zur Körperpflege genügen. Eine Möglichkeit zum Wechseln der Kleidung bestand ebensowenig wie eine Waschgelegenheit. Erschwert wurden die Verbältnisse noch besonders dadurch, daß der Angekl. ab November 1994 wieder unter starken Rückenschmerzen litt. Er konnte sich zeitweise nicht aufrichten und mußte gebückt gehen. Eine medizinische Betreuung erfolgte nicht. Stattdessen reizte der Zustand des Angekl. das Aufsichtspersonal dazu, ihn körperlich zu mißhandeln, weil sie ibn für einen Simulanten bielten. Nach seiner Verurteilung wurde der Angekl. zuerst für kurze Zeit im Hochsicherheitstrakt der Strafvollzugsanstalt untergebracht, was üblich ist. Danach kam er in den Block für Ausländer. Dort befand er sich mit 16 anderen erwachsenen Gefangenen der unterschiedlichsten Nationalitäten in einer 25 Quadratmeter großen Zelle; als Abort diente auch bier ein in der Zelle aufgestellter Eimer. Die Benutzung einer außerbalb der Zelle gelegenen Toilette wurde nur gelegentlich und dem Anschein nach willkürlich bewilligt oder verweigert. [...]."

1:2,5 Brasilien (LG München II, Urt. v. 27.01.2000 - 1 KLs 48 Js 39789/95 = StV 2001, 19)

Im Abdruck des „Strafverteidigers“ wird mitgeteilt:

"Die Zeit in brasilianischer Auslieferungshaft ist mit einem Umrechnungsfaktor von 2,5 anzurechnen, wenn der Beschuldigte in einer Zelle mit 9 qm freier Fläche, die für 6 Personen ausgelegt ist, mit 14 bis 17 Mitinsassen untergebracht wurde, als Toilette ein Loch im Boden diente, die Zelle fensterlos und obne Frischluft war, die Temperatur zeitweise bei 40 Grad Celcius lag und das verabreichte Essen unzureichend und teilweise verdorben war."

1:3 Spanien (LG Bremen, Urt. v. 13.12.1991 - 14 KLs 501 Js 6582/89 = StV 1992, 326)

„Er [der Angeklagte] war in einem heruntergekommenen Gebäude in einer Sammelzelle mit insgesamt ca. 100 Personen untergebracht. In dieser Zelle befand sich kein Tisch und kein Stubl. Als Betten dienten Holzverschläge obne Matratzen. Die Zelle war ausgestattet mit einer einzigen Toilette in Form eines in den Boden eingelassenen Lochs. Die Zelle war stark verschmutzt und von Ungeziefer wie Kakerlaken und Wanzen befallen. Auch wäbrend seiner Haft wurde er sowobl von seinen türkischen Landsleuten, die zur türkischen Abnehmergruppe gehörten, als auch vom Wachpersonal körperlich mißhandelt.[...]."

1:3 Mazedonien (LG Verden an der Aller, Urt. v. 06.07.2006 - 2-1/06 = StV 2007, 362)

„Der Angekl. teilte seine Zelle zunächst mit zwei, nach einer Woche mit einem weiteren Häftling. Den Häftlingen stand ca. 3 qm Raum zur Verfügung. Zugang zu natürlichem Licht bestand nicht, das Licht in den Zellen reichte zum Lesen nicht aus. Eine Frisch- 
luftzirkulation war nicht gegeben. Medizinisches Personal war in der JVA nicht vorbanden. [...]

Bei der Bemessung des Anrechnungsfaktors von 1:3 hat das Gericht berücksichtigt, daß dem Angekl. mit ca. 3 qm nur wenig Raum zur Verfügung stand, er zudem die sehr kleine Zelle mit zumindest einem weiteren Häftling teilen mußte und die Zelle nicht mit Zugang zu Tageslicht und Frischluft versehen war. Bereits diese Bedingungen weichen von in Deutschland erlittener U-Haft ab. Soweit das Auswärtige Amt [...] allgemein ausgeführt hat, daß bei einer Untersuchung der JVA in Obrid keine gravierenden Mängel festgestellt werden konnten, reicht die konkrete Auflistung und Beschreibung der Haftumstände im Einzelnen in diesem Schreiben nicht aus, um die Schilderung des Angekl. von den tatsächlichen Bedingungen zu widerlegen. So verbält sich die Schilderung z.B. nicht zu den bygienischen Bedingungen in derJVA und zu der tatsächlichen Erreichbarkeit von medizinischem Personal."

\section{1:2 Slovakei (LG Kleve, Beschl. v. 26.11.2002 - 2 StVK 393/02 = StV 2003, 453)}

Die Entscheidung des LG Kleve hebt sich ab von den vorgenannten, weil die geschilderten Umstände eigentlich einen Maßstab von 1:3 nahegelegt hätten. Die Gegebenheiten des Einzelfalles sowie vielleicht eine Art „vorweggenommener“ EU-Bonus heben diesen Fall von den anderen ab:

„Am 07.06.2002 sei er nach Leopoldov, eine "Festung " mit Gebäuden aus dem Jabre 1723 gebracht worden. Zunächst sei er in einer Kellerzelle gewesen, die tropfnaß und extrem kalt gewesen sei. Er habe sich einen Hexenschuß zugezogen, keine ärztliche Versorgung erhalten, sei zwei Wochen bewegungsunfähig gewesen. Die stinkenden Matratzen bätten auf dem blanken Boden gelegen. Die WCs seien unbenutzbar, stark unhygienisch gewesen. Die Fenster seien mit durchlöchertem Blech und Elektroden aus der Nazizeit gesichert gewesen, es habe ständiges Halbdunkel geherrscht. Kakerlaken und Läuse habe es in den Zellen gegeben. Duschen sei zeitlich eingeschränkt selten bis gar nicht möglich gewesen. Als Folge seien Hautkrankheiten allgemein verbreitet gewesen.

Die Essensversorgung sei mangelhaft gewesen. [...] Der Hofgang habe in einer Art »Betonschlauch « von etwa $8 \mathrm{~m}$ Länge, 1,5 $\mathrm{m}$ Breite mit einer Decke aus Stablgitter für 12 Personen zugleich stattgefunden, so daß keine Bewegung möglich gewesen sei. Rauchen sei bei Androbung von Bunkerhaft verboten gewesen. Die ständig verstopften Toiletten seien unbrauchbar gewesen. Toilettenpapier habe es nur durch übertenerten Einkauf gegeben. Unter den Gefangenen habe es ständig Übergriffe gegeben, die vom Personal »übersehen " worden seien. [...]

Zugezogene Krankheiten und körperliche Leiden: Infektion an den Füßen/Wasserblasen (Salbe Kuterid), Ischiasnerv/Hexenschuß, unbehandelt, später auf Umwegen Novalgin erhalten, Durchfall, keine Behandlung (»haben alle hier"), Kopfschmerzen bei Tageslicht oder elektrischem Licht infolge permanenter Dunkelheit, Medikamente, Toilettenpapier mußten selber bezablt werden. (...) 
Der Verurteilte hinterließ bei seiner Anbörung einen seriösen Eindruck, auch wenn er bei der Einreise in die Slowakei einen gefälschten Paß benutzte. Seine Darstellung der Verbältnisse in den slowakischen Gefängnissen bzw. Transports ab dem 06.06.2002 erscheint glaubhaft. Der zuständigen Vizekonsulin hatte er zwar keine Beschwerden mitgeteilt, wobl aber hatte er um Besuch gebeten, obwobl er eine Betreunng sonst nicht gewünscht hat. Seine Einlassung überzengt, wegen der ungünstigen Verbältnisse in den Gefängnissen sei eine offene schriftliche Beschwerde nicht tunlich gewesen, darum babe er um Besuch gebeten. Auch die Vizekonsulin äußerte - vorsichtig -, daß ein Anrechnungsmaßstab von 1:1,5 - 2 in Betracht kommen könne. Aus dieser Einschätzung ist zu schließen, daß die allgemeinen Verhältnisse in den Gefängnissen, in denen der Verurteilte ab dem 06.06.2002 in der Slowakei gewesen ist, sich von den Verbältnissen deutscher Gefängnisse erbeblich nachteilig unterscheiden. Dann aber gewinnt die Darstellung des Verurteilten Überzengungskraft. Für die Angemessenheit eines höheren Maßstabs reichen die von ihm beschriebenen erlittenen Erschwernisse nicht, auch wenn berücksichtigt wird, daß er zeitweilig bettlägerig gewesen ist und Medikamente sich auf eigene Kosten beschaffen mußte."

1:2 Weißrussland (LG Ulm, Urt. v. 12.05.2010 - 1 KLs 14 Js 24481/07 = StV 2010, 527, mit einer Anmerkung der Redaktion)

„Das Urteil hat folgende Feststellungen zu den Bedingungen der Haft getroffen: Die Haftbedingungen »waren gekennzeichnet durch Überbelegung der Zellen, feblende Hygiene und fehlende medizinische Versorgung. Die Zellen waren mangelhaft ausgestattet und zeitweilig obne Beheizung. Fenster waren trotz starker Minustemperaturen zum Teil nicht vorhanden. Tätliche Übergriffe von Wachpersonal kamen vor, Besuche von Angehörigen der deutschen Botschaft wurden überwacht."

Wie die zuvor erwähnte Entscheidung des LG Kleve fügt sich auch diese Entscheidung zu Weißrussland nicht in die übrigen ein. Aus den 90er Jahren des 20. Jahrhunderts gibt es jedoch auch die oben erwähnten Entscheidungen zu Gefängnissen in Frankreich (Lyon bzw. Montpellier), die offensichtlich baulich sehr alte Anstalten eines zentralen EUStaates betrafen, bzw. in Italien (6 Personen in einer Einmann-Zelle bei großer Hitze). Dort wurde jeweils der Maßstab 1:2 angewendet. Dies ist aus meiner Sicht nur mit einer Art „EU-Bonus“ dieser Staaten zu erklären, obwohl die geschilderten Umstände sehr vergleichbar sind mit den Fällen, die einhellig mit 1:3 bewertet worden sind.

Insgesamt kann man die deutsche Rechtsprechung charakterisieren als ein vorsichtiges Herantasten an höhere Maßstäbe als 1:1. Letztlich sind es im Ergebnis die vom EGMR und auch schon vom Bundesverfassungsgericht aufgezeigten Faktoren - vor allem der Raumgröße im Verhältnis zur Insassenzahl, welche letztlich die bisherige Praxis mit bestimmt haben.

Zwar legen die Fälle, in denen die deutsche Rechtsprechung den Maßstab 1:3 bejaht hat, auch eine Verletzung des Art. 3 EMRK nahe. Sind die Kriterien des EGMR für dessen 
unwiderlegliche Bejahung jedoch eindeutig erfüllt, so müsste die deutsche Praxis konsequenterweise auch den Maßstab 1:4 anwenden.

\section{Zusammenfassung}

1. $\ 51$ Abs. 4 S. 2 StGB ist eine gesetzgeberische Entscheidung für eine Anrechnungslösung gerade auch bei menschenrechtswidriger ausländischer Haft und gegen eine bloße Strafzumessungslösung.

2. Die deutsche Praxis zu $\ 51$ Abs. 4 S. 1 reicht von 1:1-Fällen (z. B. EU-Staaten) bis zu 1:3-Anrechnungen in gravierenden Fällen.

3. Nach der EGMR-Praxis führen bestimmte Kriterien unwiderleglich zu einer Bejahung von Art. 3 EMRK (unmenschliche Behandlung). Auch weil Art. 3 notstandsfest ist (Art. 15 EMRK), liegt eine weitere Kategorie (1:4-Anrechnung) mehr als nahe, wenn die EGMR-Kriterien erfüllt sind.

Literatur:

Frank (1931) Das Strafgesetzbuch für das Deutsche Reich, 18. Aufl.

Heintschel-Heinegg (2012) Beck'scher Online-Kommentar StGB

Höpfel / Ratz (Hrsg.) (2011) Wiener Kommentar zum StGB, 2. Aufl.

Karpenstein / Mayer (2012) EMRK. Kommentar

Kindbäuser / Neumann / Paeffgen (2013) Nomoskommentar StGB, Band 1, 4. Aufl.

Satzer / Schmitt / Widmaier (2009) Strafgesetzbuch Kommentar

Schomburg / Lagodny / Gleß / Hackner (2012) Internationale Rechtshilfe in Strafsachen, 5. Aufl.

Schwartz (1914) Das Strafgesetzbuch für das Deutsche Reich von Olshausen (1892) Kommentar zum Strafgesetzbuch für das Deutsche Reich, 4. Aufl. von Olshausen (1914) Kommentar zum Strafgesetzbuch für das Deutsche Reich, 12. Aufl.

Kontakt:

Univ. Prof. Dr. Otto Lagodny

Universitätsprofessor für österreichisches und ausländisches

Straf- und Strafverfabrensrecht, sowie Strafrechtsvergleichung

Paris Lodron Universität Salzburg

Kapitelgasse $5-7$

5020 Salzburg

Otto.Lagodny@sbg.ac.at.

NK 26. Jg. 3/2014 